\title{
Terapia miofuncional: tratamiento alternativo para corregir mordida cruzada anterior en 15 días en un paciente de 8 años de edad. Reporte de un caso
}

\section{Myofunctional therapy: alternative treatment to correct anterior crossbite in 15 days in an 8-year-old patient. Report of a case}

\section{Samy Samay Vinueza Jarrín ${ }^{1}$ José Miguel Pinto ${ }^{2}$ Nathaly Chávez Jaramillo ${ }^{3}$}

'Universidad San Francisco de Quito, Colegio de Ciencias de la Salud, Escuela de Odontología, Clínica Odontológica, Cumbayá, oficina C0 106, casilla postal 17-1200-841. Quito- Ecuador. Correo electrónico: svinuezaj@estud.usfq.edu.ec

${ }^{2}$ Universidad San Francisco de Quito, Colegio de Ciencias de la Salud, Escuela de Odontología, Clínica Odontológica, Cumbayá, oficina C0 106, casilla postal 17-1200-841. Quito- Ecuador. Correo electrónico: jmpinto@usfa.edu.ec ${ }^{3}$ Universidad San Francisco de Quito, Colegio de Ciencias de la Salud, Escuela de Odontología, Clínica Odontológica, Cumbayá, oficina C0 106, casilla postal 17-1200-841. Quito- Ecuador. Correo electrónico: nchavezj@asig.com.ec.

Editado por / Edited by: Johanna Monar

Recibido / Received: 19-11-2020

Aceptado / Accepted: 13-09-2021

Publicado en linea / Published online:09/2021 


\section{Resumen}

La mordida cruzada anterior es una maloclusión cada vez más observable en pacientes pediátricos en desarrollo. La comprensión y análisis de sus factores etiológicos es esencial para la búsqueda de un tratamiento apropiado, cómodo y eficaz para redirigir el patrón de crecimiento anormal. Es así que, el presente artículo reporta el caso clínico de una paciente de sexo femenino de 8 años 10 meses de edad. La misma, posterior a la evaluación clínica y realización de exámenes complementarios, recibió el diagnóstico de mordida cruzada anterior asociada a deglución atípica. Frente a esta situación, se propuso como tratamiento el establecimiento de terapia miofuncional oral, la cual se manifiesta como una alternativa terapéutica única y efectiva para devolver la estética y función.

Palabras clave: Terapia miofuncional, Mordida cruzada anterior, Mordida invertida, Deglución atípica, Odontopediatría.

\section{Abstract}

Anterior crossbite is an increasingly observable malocclusion in developing pediatric patients. Their understanding and analysis of etiological factors is essential for the search for an appropriate, comfortable and effective treatment to redirect the abnormal growth pattern. Thus, this article reports the clinical case of an 8-year-10month-old female patient. After the clinical evaluation and complementary tests, the patient received the diagnosis of anterior crossbite associated with atypical swallowing. Faced with this situation, the establishment of oral myofunctional therapy is proposed as a treatment, which is manifested as a unique and effective therapeutic alternative to restore aesthetics and function.

Keywords: Myofunctional therapy, Anterior crossbite, Inverted bite, Atypical deglution, Pediatric dentistry. 


\section{Introducción}

La maloclusión es definida como una condición caracterizada por relaciones anormales entre las unidades dentarias ${ }^{1}$ representa el punto final, clínicamente observable, de numerosas influencias genéticas y ambientales ${ }^{2,3}$. Un ejemplo claro de dicha expresión es, sin duda, la mordida cruzada anterior, que se presenta con una prevalencia entre el 2,2 al 36\% ${ }^{4,5}$ y afecta en gran medida a pacientes pediátricos en desarrollo, sobre todo en etapa de dentición mixta ${ }^{5}$. Esta afección del desarrollo implica una posición lingual de incisivos y/o caninos superiores en relación a sus homólogos inferiores como resultado de una alteración en los componentes dentales, funcionales o esqueléticos del sistema estomatognático ${ }^{4-7}$.

Como mecanismo de prevención del crecimiento aberrante de estos componentes, la mordida cruzada anterior debe ser corregida tan pronto se detecte y se obtenga la colaboración del niño y de sus padres/representantes ${ }^{4-6}$. De esta manera; dependiendo de la etiología, edad, cantidad de dientes afectados y grado de motivación, la literatura presenta múltiples opciones de tratamiento temprano que varían desde aparatos ortopédicos fijos o removibles, planos inclinados de mordida, terapia miofuncional y una combinación de aquellos ${ }^{4,5}$.

La Terapia Miofuncional Oral, introducida principalmente por Rogers a principios de la década de $1900^{7}$, se basa en ejercicios destinados a proporcionar un equilibro funcional a través de la reeducación del patrón neuromuscular para corregir posturas funcionales y de reposo anormales ${ }^{2,3,8-10}$. Aunque su concepto ha sido discutido y ha generado un gran debate desde esta época, los beneficios para el tratamiento de mal oclusiones, en particular de mordida cruzada anterior, no ha sido abordada ampliamente en publicaciones científicas ${ }^{2,3}$.

Es por ello, que la finalidad de este artículo es presentar a la terapia miofuncional como una alternativa terapéutica única y efectiva en pacientes colaboradores que presentan una mordida cruzada anterior dental a través de la presentación de un caso clínico.

\section{Presentación de caso clínico}

Paciente femenino de 8 años 10 meses de edad asiste a la Clínica Odontológica de Posgrado de la Universidad San Francisco de Quito (USFQ), refiriendo como motivo de consulta "El problema es la mordida". Sin presencia de síntomas y acompañada de su padre, en el interrogatorio se señala como antecedentes personales de la paciente: reacciones alérgicas al polvo y animales y, su historial familiar denota predisposición genética a presentar hipertensión.

Al examen clínico extraoral, la paciente presenta un biotipo mesofacial, perfil recto, proporciones faciales simétricas con coincidencia de la línea media facial y dental, ángulos nasolabial y nasomental en la norma. Por otro lado, al análisis intraoral se evidencia las siguientes características: arcada superior e inferior de forma 
cuadrada, ligero apiñamento en unidades dentarias ántero-inferiores, clase molar y canina I. Además, la presencia de mordida cruzada anterior involucrando a los incisivos centrales superiores e inferiores (OB: -2mm; OJ: - 1mm), deglución atípica al análisis funcional, así como también opacidades blanco-amarillentas y marrones en varias unidades dentarias. Como herramientas auxiliares para el establecimiento diagnóstico se utilizaron registros fotográficos extra e intraorales, modelos de estudio y una radiografía panorámica. Estos dos últimos, fueron fundamentales para predecir que no existe una falta de espacio en las arcadas para la subsecuente erupción dentaria.

El conjunto de hallazgos clínicos coincidentes y en complemento con los encontrados en las distintas herramientas auxiliares permiten llegar al diagnóstico de mordida cruzadaanterior de tipo dentalasociadaa degluciónatípicaasícomo hipomineralización de segundos molares primarios e hipomineralización molar-incisivo para la dentición permanente. Es importante mencionar que, durante todo este proceso de evaluación resalta la gran colaboración y preocupación del representante así como de la paciente, lo cual permite catalogarla como definitivamente positiva (++) en la escala de Frankl. Esta condición favorable para la atención y éxito clínico en odontopediatría; así como, su edad y la etiología de la maloclusión nos llevan a considerar a la terapia miofuncional como opción terapéutica para el caso.

Los ejercicios empleados consistieron en: realizar sonidos semejantes a un sapo cuando croa, pronunciar palabras con los fonemas r, s, t, c, f con la boca cerrada, deslizar la lengua por el paladar de adelante hacia atrás con los dientes en oclusión y de la misma manera por las caras palatinas/ linguales de las unidades dentarias. Como indicación, estos ejercicios debían ser alternados y realizados durante todo el día con una duración mínima de 30 minutos en total. Para ello, se educa al padre y a la paciente acerca de la importancia de la constancia y buena ejecución de los mismos. Quince días después de la aceptación e instauración del tratamiento, se realiza el primer control en el cual se observa el descruzamiento de la mordida invertida. Finalmente, con el objetivo de mejorar la relación oclusal a nivel anterior, la terapia miofuncional continúa por 3 meses con disminución en la frecuencia de los ejercicios de forma secuencial hasta su suspención. A los 6 meses de seguimiento y control, no se evidenció recidiva de la maloclusión inicial.

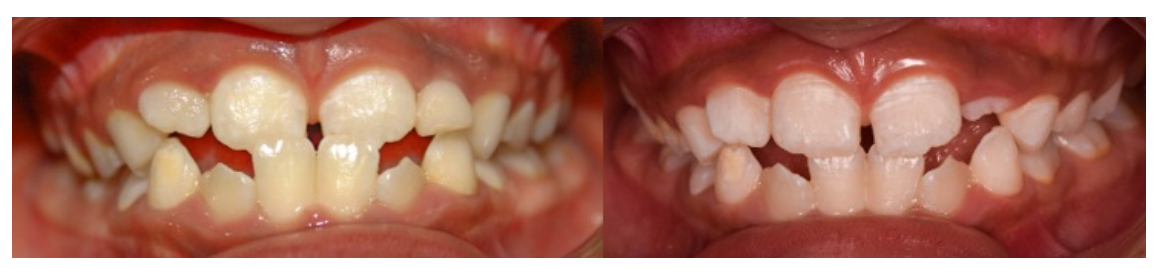

Figura 1. Fotografía intraoral de frente. a) Inicial. b) Control a los 15 dias. 


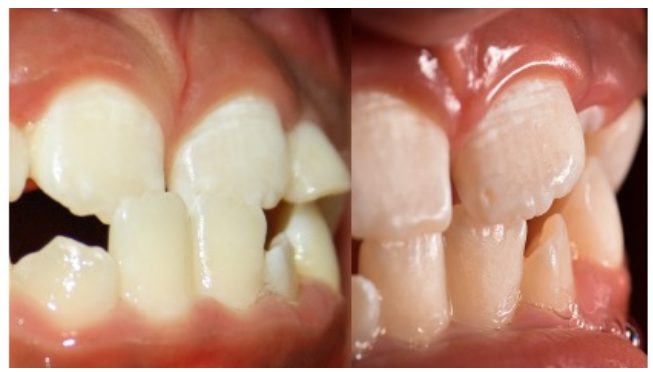

Figura 2. Fotografía intraoral de sobremordida. a) Inicial. b) Control a los 15 días.

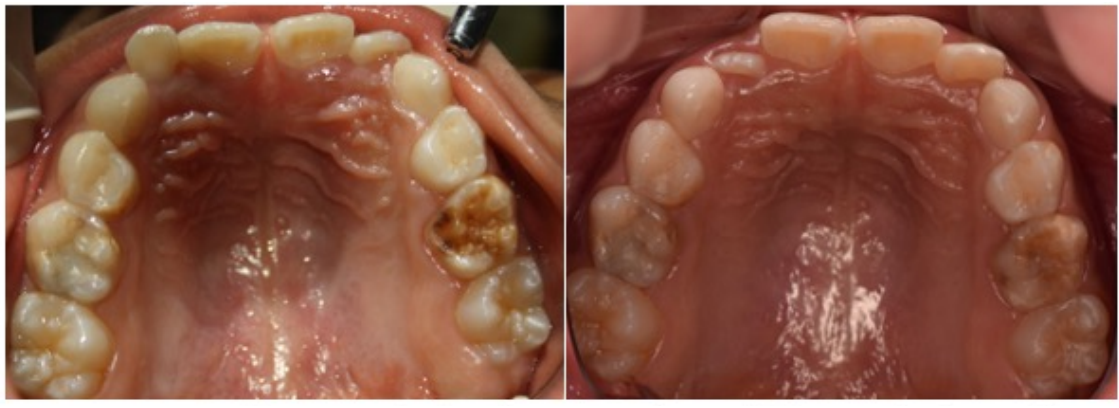

Figura 3. Fotografía intraoral oclusal superior. a) Inicial. b) Control a los 15 días.

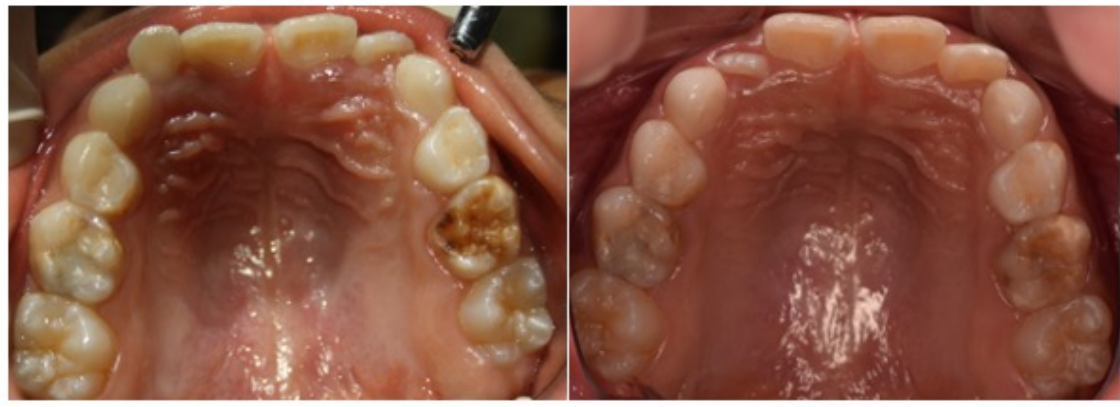

Figura 4. Fotografía intraoral oclusal inferio. a) Inicial. b) Control a los 15 días.

\section{Discusión}

Una mordida cruzada anterior de tipo dental puede ser definida como aquella en la que se involucra 1 o 2 dientes, en los cuales, los incisivos superiores adoptan una posición 
más retroinclinada en relación a los incisivos mandibulares ${ }^{6}$. Esta maloclusión si no es interrumpida lo antes posible, tiene un efecto desfavorable sobre el patrón de crecimiento, porque puede conducir al desarrollo de un problema esquelético'. En esta condición, la mandíbula ralentiza el crecimiento del maxilar superior y su posición avanzada favorece el crecimiento hacia adelante ${ }^{11}$. Además, su tratamiento temprano nos ayuda a prevenir el desgaste y fracturas a nivel dentario, problemas endo-periodontales y trastornos témporo-mandibulares ${ }^{5}$.

La etiología de una mordida cruzada anterior es multifactorial; sin embargo, la alta prevalencia de su asociación con patrones de movimientos compensatorios, también permite considerar dentro del análisis funcional la presencia de una deglución atípica ${ }^{12}$. Este trastorno puede ser definido como una disfunción oral que se genera cuando no se produce la correcta maduración de la deglución y las características típicas de la deglución infantil, como el empuje de la lengua, persisten incluso después de los 6 años ${ }^{11,12}$. Producto de este patrón inadecuado, se realiza un esfuerzo perioral excesivo y la lengua ejerce presión hacia adelante y de manera lateral en las unidades dentarias, lo cual contribuye a la presencia de la maloclusión ${ }^{13}$.

Bajo este punto de vista, frente al diagnóstico de una mordida cruzada anterior con presencia de deglución atípica y la notable colaboración del paciente, el tratamiento inicial en el caso presentado considera a la terapia miofuncional como el mecanismo ideal que permita la reposición de la postura anormal de la lengua y consecuentemente de los órganos dentarios. De esta manera, bajo este enfoque conservadory, no costoso, podemos ver como la relación intermaxilar y de posición, así como la angulación de los incisivos involucrados mejoraron notablemente en un corto período de tiempo.

Aunque, como vemos el manejo de un tratamiento miofuncional en niños con dentición mixta posee un enfoque prometedor, la calidad de la evidencia existente es insuficiente con respecto a su aplicación en maloclusiones ${ }^{3}$. La mayoría de estudios respaldan su uso en casos de mordidas abiertas de tipo anterior asociados a deglución atípica ${ }^{9,10,12,14}$ o apnea obstructiva del sueño², sobre todo como complemento de la terapia ortodóntica. Sin embargo, la evidencia en casos de mordida cruzada anterior con asociación a deglución atípica es totalmente limitada y más aún si la llegamos a considerar como mecanismo de tratamiento único. En este sentido, Begnoni" es el único autor que, bajo un diseño de estudio longitudinal, demuestra la efectividad de esta terapia para establecer un patrón de activación muscular fisiológica.

Finalmente, es imperativo el desarrollo de más investigaciones para comprender los factores que injieren en el éxito del tratamiento, con el fin de definir mejor el momento y los criterios para seleccionar a los pacientes adecuados. Es por esta razón, que el caso abordado es crucial, debido a que demuestra el éxito clínico de la terapia miofuncional y expone a la colaboración del paciente y padres de familia como un factor previo a considerar. 


\section{Conclusión}

Uno de los principales propósitos de la Odontopediatría es prevenir y corregir potenciales maloclusiones facilitando el desarrollo de los maxilares y el correcto posicionamiento de los dientes. Para los niños en período de crecimiento, la resolución de este tipo de problemas con intervenciones menores representa un mecanismo favorable que afecta positivamente su vida social y autoestima. En este sentido, el tratamiento de mordida cruzada anterior con asociación a deglución atípica a través de terapia miofuncional es un enfoque conservador, eficaz, económico y de corto plazo cuando se diagnostica correctamente y cuenta con la colaboración de padres y pacientes. 


\section{Bibliografía}

1. Pellegrino M, Caruso S, Cantile T, Pellegrino G, Ferrazzano GF. Early treatment of anterior crossbite with eruption guidance appliance: A case report. International Journal of Environmental Research and Public Health. 2020;17(10). doi:10.3390/ijerph17103587

2. Wishney M, Darendeliler MA, Dalci O. Myofunctional therapy and prefabricated functional appliances: an overview of the history and evidence. Australian Dental Journal. 2019;64(2):135144. doi:10.1111/adj.12690

3. Koletsi D, Makou M, Pandis N. Effect of orthodontic management and orofacial muscle training protocols on the correction of myofunctional and myoskeletal problems in developing dentition. A systematic review and meta-analysis. Orthodontics and Craniofacial Research. 2018;21(4):202-215. doi:10.1111/ocr.12240

4. Khalaf K, Mando M. Removable appliances to correct anterior crossbites in the mixed dentition: a systematic review. Acta Odontologica Scandinavica. 2020;78(2):118-125. https://doi.org/10.1 080/00016357.2019.1657178. doi:10.1080/00016357.2019.1657178

5. Canan Akdik DC. Taking a Giance At Anterior Crossbite in Children: Case Series. Contemporary Clinical Dentistry. 2017;8(September):11-9. doi:10.4103/ccd.ccd

6. Jorge JO, Corradi-Dias L, Flores-Mir C, Pordeus IA, Paiva SM, Abreu LG. Comparison Between Removable and Fixed Devices for Nonskeletal Anterior Crossbite Correction in Children and Adolescents: A Systematic Review. Journal of Evidence-Based Dental Practice. 2020;20(3):101423. https://doi.org/10.1016/j.jebdp.2020.101423. doi:10.1016/j. jebdp.2020.101423

7. Papageorgiou SN, Koletsi D, Eliades T. What evidence exists for myofunctional therapy with prefabricated appliances? A systematic review with meta-analyses of randomised trials. Journal of Orthodontics. 2019. doi:10.1177/1465312519880558

8. Tanny L, Huang B, Naung NY, Currie G. Non-orthodontic intervention and non-nutritive sucking behaviours: A literature review. Kaohsiung Journal of Medical Sciences. 2018;34(4):215-222. https://doi.org/10.1016/j.kjms.2018.01.006. doi:10.1016/j.kjms.2018.01.006

9. Van Dyck C, Dekeyser A, Vantricht E, Manders E, Goeleven A, Fieuws S, Willems G. The effect of orofacial myofunctional treatment in children with anterior open bite and tongue dysfunction: A pilot study. European Journal of Orthodontics. 2016;38(3):227-234. doi:10.1093/ejo/cjv044

10. Degan VV, Puppin-Rontani RM. Remoção de hábitos e terapia miofuncional: restabelecimento da deglutição e repouso lingual. Pró-Fono Revista de Atualização Científica. 2005;17(3):375382. doi:10.1590/s0104-56872005000300011

11. Begnoni G, Dellavia C, Pellegrini G, Scarponi L, Schindler A, Pizzorni N. The efficacy of myofunctional therapy in patients with atypical swallowing. European Archives of OtoRhino-Laryngology. 2020;277(9):2501-2511. https://doi.org/10.1007/s00405-020-05994-w. doi:10.1007/s00405-020-05994-w

12. Di Vecchio S, Manzini P, Candida E, Gargari M. Froggy mouth: A new myofunctional approach to atypical swallowing. European Journal of Paediatric Dentistry. 2019;20(1):33-37. doi:10.23804/ ejpd.2019.20.01.07

13. D'Onofrio L. Oral dysfunction as a cause of malocclusion. Orthodontics and Craniofacial Research. 2019;22(S1):43-48. doi:10.1111/ocr.12277

14. Rivero CM. Manejo De Una Mordida Abierta Anterior Con Deglución Disfuncional Mediante Ortopedia Funcional De Los Maxilares Con Sn3: Reporte De Un Caso. Ustasalud Odontología. 2006;5:64-76. http://scholar.google.com/ scholar?hl=en\&btnG=Search\&q=intitle:No+Title\#0\%5Cnhttp://200.21.227.151/inicio/ publicaciones/div_salud/pdf/volumen_5_(1).pdf\#page=64 